\title{
Stochastic Signal Processing for Sound Environment System with Decibel Evaluation and Energy Observation
}

\author{
Akira Ikuta and Hisako Orimoto \\ Department of Management Information Systems, Prefectural University of Hiroshima, Hiroshima 734-8558, Japan \\ Correspondence should be addressed to Akira Ikuta; ikuta@pu-hiroshima.ac.jp
}

Received 21 April 2014; Accepted 3 June 2014; Published 24 July 2014

Academic Editor: Suiyang Khoo

Copyright ( 2014 A. Ikuta and H. Orimoto. This is an open access article distributed under the Creative Commons Attribution License, which permits unrestricted use, distribution, and reproduction in any medium, provided the original work is properly cited.

In real sound environment system, a specific signal shows various types of probability distribution, and the observation data are usually contaminated by external noise (e.g., background noise) of non-Gaussian distribution type. Furthermore, there potentially exist various nonlinear correlations in addition to the linear correlation between input and output time series. Consequently, often the system input and output relationship in the real phenomenon cannot be represented by a simple model using only the linear correlation and lower order statistics. In this study, complex sound environment systems difficult to analyze by using usual structural method are considered. By introducing an estimation method of the system parameters reflecting correlation information for conditional probability distribution under existence of the external noise, a prediction method of output response probability for sound environment systems is theoretically proposed in a suitable form for the additive property of energy variable and the evaluation in decibel scale. The effectiveness of the proposed stochastic signal processing method is experimentally confirmed by applying it to the observed data in sound environment systems.

\section{Introduction}

A specific signal in real sound environment system usually exhibits multifarious and complex characteristics such as non-Gaussian distribution and nonlinear property relating to natural, social, or human factors. Furthermore, the observation data usually are contaminated by external noise (e.g., background noise) with complex statistical properties. In this situation, in order to evaluate the sound environment system, precise estimation of the system characteristics of the sound environment is required by considering the contaminated observation data.

Furthermore, the internal physical mechanism of the real sound environment system is often difficult to recognize analytically, and it contains unknown structural characteristics. In our previous study, it was found that complex sound environment systems are difficult to analyze by using usual structural methods based on the physical mechanism [1]. Therefore, a nonlinear system model was derived in the expansion series form reflecting various types of correlation information from the lower order to the higher order between input and output variables [2]. The conditional probability density function contains the linear and nonlinear correlations in the expansion coefficients and these correlations play an important role as the statistical information for the input and output relationship of sound environment system.

On the other hand, in considering the relationship between the evaluation from top-down viewpoint and the countermeasure from bottom-up viewpoint in the sound environment system, noise evaluation quantities in decibel scale like $L_{x}\left((100-x)\right.$ percentile level) and $L_{\text {Aeq }}$ (averaged energy on decibel scale) and some countermeasure methods in energy scale are widely used. Since there is a certain scale transform between decibel and energy variables, a unified general consideration without losing their mutual relationship has to be derived.

In this study, a general type of complex sound environment systems is considered. A stochastic signal processing method for predicting the output response probability distribution in decibel scale based on the input observations is proposed for complex sound environment systems. 
More specifically, an expansion expression of the conditional probability distribution in decibel scale is adopted as the system characteristics. Next, a method to estimate the system parameters reflecting several orders of correlation information between the input and output variables is derived by considering the additive property of energy variables under existence of external noise. Furthermore, a prediction method for the output probability distribution in decibel scale is also considered.

The effectiveness of the proposed theory is confirmed experimentally by applying it to real data of a sound insulation system and the road traffic noise environment measured around a national road in Hiroshima city.

\section{Evaluation of Sound Environment System under Existence of External Noise}

2.1. Statistical Model for Sound Environment System. Let $X$ and $Y$ be the sound pressure levels of input and output signals for a complex sound environment system. The probability distribution of output $Y$ has to be predicted on the basis of the observed data of the input level $X$, because noise evaluation quantities connected with probability distribution are widely used. All the information on linear and/or nonlinear correlations between $X$ and $Y$ is included in the conditional probability density function $P(Y \mid X)$ [2].

In order to find explicitly the various correlation properties between $X$ and $Y$, let us expand the joint probability density function $P(X, Y)$ into an orthogonal polynomial series [3], as follows:

$$
\begin{gathered}
P(X, Y)=P_{0}(X) P_{0}(Y) \sum_{r=0}^{\infty} \sum_{s=0}^{\infty} A_{r s} \varphi_{r}^{(1)}(X) \varphi_{s}^{(2)}(Y), \\
A_{r s} \equiv\left\langle\varphi_{r}^{(1)}(X) \varphi_{s}^{(2)}(Y)\right\rangle
\end{gathered}
$$

where \langle\rangle denotes the averaging operation. $P_{0}(X)$ and $P_{0}(Y)$ can be chosen arbitrarily as the probability density functions describing the dominant parts of the actual fluctuation pattern. Two functions $\varphi_{r}^{(1)}(X)$ and $\varphi_{s}^{(2)}(Y)$ are orthogonal polynomials with the weighting functions $P_{0}(X)$ and $P_{0}(Y)$. The information on the various types of linear and/or nonlinear correlations between $X$ and $Y$ is reflected hierarchically in each expansion coefficient $A_{r s}$. In this section, the Gaussian distribution suitable for the random variables in decibel scale is adopted as $P_{0}(X)$ and $P_{0}(Y)$

$$
\begin{gathered}
P_{0}(X)=N\left(X ; \mu_{X}, \sigma_{X}^{2}\right), \\
P_{0}(Y)=N\left(Y ; \mu_{Y}, \sigma_{Y}^{2}\right)
\end{gathered}
$$

with

$$
\begin{array}{cc}
N(x ; \mu, \sigma) \equiv \frac{1}{\sqrt{2 \pi \sigma^{2}}} e^{-(x-\mu)^{2} / 2 \sigma^{2}}, \\
\mu_{X} \equiv\langle X\rangle, \quad \sigma_{X}^{2} \equiv\left\langle\left(X-\mu_{X}\right)^{2}\right\rangle, \\
\mu_{Y} \equiv\langle Y\rangle, \quad \sigma_{Y}^{2} \equiv\left\langle\left(Y-\mu_{Y}\right)^{2}\right\rangle .
\end{array}
$$

Thus, orthogonal polynomials $\varphi_{r}^{(1)}(X)$ and $\varphi_{s}^{(2)}(Y)$ are given by the Hermite polynomial [3]:

$$
\begin{gathered}
\varphi_{r}^{(1)}(X)=\frac{1}{\sqrt{r !}} H_{r}\left(\frac{X-\mu_{X}}{\sigma_{X}}\right), \\
\varphi_{s}^{(2)}(Y)=\frac{1}{\sqrt{s !}} H_{s}\left(\frac{Y-\mu_{Y}}{\sigma_{Y}}\right) .
\end{gathered}
$$

Substituting (1) into the definition of the conditional probability, $P(Y \mid X)$ can be expressed in an expansion series form as follows:

$$
\begin{aligned}
P(Y \mid X) & =\frac{P(X, Y)}{P(X)} \\
& =\frac{P_{0}(Y) \sum_{r=0}^{\infty} \sum_{s=0}^{\infty} A_{r s} \varphi_{r}^{(1)}(X) \varphi_{s}^{(2)}(Y)}{\sum_{r=0}^{\infty} A_{r 0} \varphi_{r}^{(1)}(X)} .
\end{aligned}
$$

\subsection{Estimation of Correlation Information Based on Energy} Observation. In the measurement of the sound environment, the effects by external noise (e.g., background noise) are inevitable. Then, based on the additive property of energy variable, the observed sound intensity $z_{k}$ at a discrete time $k$ is expressed as

$$
z_{k}=y_{k}+v_{k}
$$

where $y_{k}$ and $v_{k}$ are sound intensities of the output signal for the sound environment system and external noise. We assume that the statistics of the external noise are known. In this section, an estimation method for the expansion coefficients $A_{r s}$ in (1), reflecting the correlation information between $X$ and $Y$, is derived on the basis of the observed data $z_{k}$. There are relationships between energy variables $x_{k}, y_{k}$ and decibel variables $X_{k}, Y_{k}$ for the input and output signals, as

$$
\begin{gathered}
X_{k}=10 \log _{10} \frac{x_{k}}{E_{0}}[\mathrm{~dB}], \quad Y_{k}=10 \log _{10} \frac{y_{k}}{E_{0}}[\mathrm{~dB}], \\
E_{0}=10^{-12}\left[\mathrm{~W} / \mathrm{m}^{2}\right] .
\end{gathered}
$$

Therefore, from (7), the following relationships can be obtained

$$
x_{k}=e^{\left(X_{k}-K\right) / C}, \quad y_{k}=e^{\left(Y_{k}-K\right) / C}
$$

with

$$
C \equiv \frac{10}{\ln 10}, \quad K \equiv-C \ln E_{0} .
$$

Next, considering the expansion coefficients $A_{r s}$ as unknown parameter vector a,

$$
\begin{gathered}
\mathbf{a} \equiv\left(a_{1}, a_{2}, a_{3}, \ldots\right) \equiv\left(\mathbf{a}_{(1)}, \mathbf{a}_{(2)}, \ldots\right), \\
\mathbf{a}_{(s)} \equiv\left(A_{0 s}, A_{1 s}, A_{2 s} \ldots\right), \quad(s=1,2, \ldots),
\end{gathered}
$$

the simple dynamical model,

$$
\begin{gathered}
\mathbf{a}_{k+1}=\mathbf{a}_{k}, \\
\left(\mathbf{a}_{k} \equiv\left(a_{1, k}, a_{2, k}, a_{3, k}, \ldots\right) \equiv\left(\mathbf{a}_{(1), k}, \mathbf{a}_{(2), k}, \ldots\right)\right),
\end{gathered}
$$


is naturally introduced for the successive estimation of the parameter.

In order to derive the estimation algorithm of the parameter, attention is focused on Bayes' theorem for the conditional probability distribution:

$$
P\left(\mathbf{a}_{k} \mid Z_{k}\right)=\frac{P\left(\mathbf{a}_{k}, z_{k} \mid Z_{k-1}\right)}{P\left(z_{k} \mid Z_{k-1}\right)}
$$

where $Z_{k} \equiv\left\{z_{1}, z_{2}, \ldots, z_{k}\right\}$ is a set of observation data up to time $k$. Based on (12), using the similar calculation process to the previously reported paper [4], the estimate for an arbitrary polynomial function $f_{\mathbf{M}}\left(\mathbf{a}_{k}\right)$ of $\mathbf{a}_{k}$ with $\mathbf{M t h}$ order can be derived as follows (cf. Appendix):

$$
\widehat{f}_{\mathbf{M}}\left(\mathbf{a}_{k}\right) \equiv\left\langle f_{\mathbf{M}}\left(\mathbf{a}_{k}\right) \mid Z_{k}\right\rangle=\frac{\sum_{\mathbf{m}=\mathbf{0}}^{\mathbf{M}} \sum_{n=0}^{\infty} B_{\mathbf{m} n} C_{\mathbf{M m}} \theta_{n}^{(2)}\left(z_{k}\right)}{\sum_{n=0}^{\infty} B_{0 n} \theta_{n}^{(2)}\left(z_{k}\right)}
$$

with

$$
B_{\mathbf{m} n} \equiv\left\langle\theta_{\mathbf{m}}^{(1)}\left(\mathbf{a}_{k}\right) \theta_{n}^{(2)}\left(z_{k}\right) \mid Z_{k-1}\right\rangle, \quad\left(\mathbf{m} \equiv\left(m_{1}, m_{2}, \ldots\right)\right) .
$$

Two functions $\theta_{\mathbf{m}}^{(1)}\left(\mathbf{a}_{k}\right)$ and $\theta_{n}^{(2)}\left(z_{k}\right)$ are orthonormal polynomials with the weighting functions $P_{0}\left(\mathbf{a}_{k} \mid Z_{k-1}\right)$ and $P_{0}\left(z_{k} \mid\right.$ $\left.Z_{k-1}\right)$. Furthermore, $C_{\mathbf{M m}}$ is the coefficient when the function $f_{\mathbf{M}}\left(\mathbf{a}_{k}\right)$ is expanded as

$$
f_{\mathbf{M}}\left(\mathbf{a}_{k}\right)=\sum_{\mathbf{m}=\mathbf{0}}^{\mathbf{M}} C_{\mathbf{M m}} \theta_{\mathbf{m}}^{(1)}\left(\mathbf{a}_{k}\right)
$$

As the concrete expression on the fundamental probability function for the parameter $\mathbf{a}_{k}$ fluctuating in both positive and negative range, a standard Gaussian distribution is adopted. Furthermore, a gamma distribution is adopted as the probability function for the sound intensity $z_{k}$ :

$$
\begin{gathered}
P_{0}\left(\mathbf{a}_{k} \mid Z_{k-1}\right)=\prod_{i} N\left(a_{i, k} ; a_{i, k}^{*}, \Gamma_{i, k}\right), \\
P_{0}\left(z_{k} \mid Z_{k-1}\right)=P_{\Gamma}\left(z_{k} ; m_{k}^{*}, s_{k}^{*}\right),
\end{gathered}
$$

with

$$
\begin{gathered}
P_{\Gamma}(x ; m, s) \equiv \frac{x^{m-1}}{\Gamma(m) s^{m}} e^{-x / s}, \\
a_{i, k}^{*} \equiv\left\langle a_{i, k} \mid Z_{k-1}\right\rangle, \quad \Gamma_{i, k} \equiv\left\langle\left(a_{i, k}-a_{i, k}^{*}\right)^{2} \mid Z_{k-1}\right\rangle, \\
m_{k}^{*} \equiv \frac{z_{k}^{* 2}}{\Omega_{k}}, \quad s_{k}^{*} \equiv \frac{\Omega_{k}}{z_{k}^{*}}, \\
z_{k}^{*} \equiv\left\langle z_{k} \mid Z_{k-1}\right\rangle, \quad \Omega_{k} \equiv\left\langle\left(z_{k}-z_{k}^{*}\right)^{2} \mid Z_{k-1}\right\rangle .
\end{gathered}
$$

Therefore, the orthogonal polynomials with the weighting functions of (16) are given by Hermite polynomial and Laguerre polynomial [3]:

$$
\begin{aligned}
& \theta_{\mathbf{m}}^{(1)}\left(\mathbf{a}_{k}\right)=\prod_{i} \frac{1}{\sqrt{m_{i} !}} H_{m_{i}}\left(\frac{a_{i, k}-a_{i, k}^{*}}{\sqrt{\Gamma_{i, k}}}\right), \\
& \theta_{n}^{(2)}\left(z_{k}\right)=\sqrt{\frac{\Gamma\left(m_{k}^{*}\right) n !}{\Gamma\left(m_{k}^{*}+n\right)}} L_{n}^{\left(m_{k}^{*}-1\right)}\left(\frac{z_{k}}{s_{k}^{*}}\right) .
\end{aligned}
$$

By considering (6) and independence of $y_{k}$ and $v_{k}$, two parameters $z_{k}^{*}$ and $\Omega_{k}$ in (17) can be given by

$$
\begin{gathered}
z_{k}^{*}=y_{k}^{*}+\left\langle v_{k}\right\rangle, \quad\left(y_{k}^{*} \equiv\left\langle y_{k} \mid Z_{k-1}\right\rangle\right), \\
\Omega_{k}=\left\langle\left(y_{k}-y_{k}^{*}\right)^{2} \mid Z_{k-1}\right\rangle+\left\langle\left(v_{k}-\left\langle v_{k}\right\rangle\right)^{2}\right\rangle .
\end{gathered}
$$

Considering (5) and the property of conditional expectation, the first terms of the right sides in the above equations are expressed as follows:

$$
\begin{aligned}
\left\langle y_{k} \mid Z_{k-1}\right\rangle & =\left\langle\left\langle y_{k} \mid x_{k}, Z_{k-1}\right\rangle \mid Z_{k-1}\right\rangle \\
& =\left\langle\int_{0}^{\infty} y_{k} P\left(y_{k} \mid x_{k}\right) d y_{k} \mid Z_{k-1}\right\rangle, \\
\left\langle\left(y_{k}-y_{k}^{*}\right)^{2} \mid Z_{k-1}\right\rangle & \\
= & \left\langle\int_{0}^{\infty} y_{k}^{2} P\left(y_{k} \mid x_{k}\right) d y_{k} \mid Z_{k-1}\right\rangle-y_{k}^{* 2} .
\end{aligned}
$$

The integrals in (20) can be calculated by using the relationship between energy and decibel variables in (8) and expansion expression in (1), as follows:

$$
\begin{aligned}
I_{l} \equiv & \int_{0}^{\infty} y_{k}^{l} P\left(y_{k} \mid x_{k}\right) d y_{k} \\
= & \int_{-\infty}^{\infty} e^{l\left(Y_{k}-K\right) / C} P\left(Y_{k} \mid X_{k}\right) d Y_{k} \\
= & \left(\int_{-\infty}^{\infty} e^{l\left(Y_{k}-K\right) / C} P_{0}\left(Y_{k}\right)\right. \\
& \left.\times \sum_{r=0}^{\infty} \sum_{s=0}^{\infty} A_{r s} \varphi_{r}^{(1)}\left(X_{k}\right) \frac{1}{\sqrt{s !}} H_{s}\left(\frac{Y_{k}-\mu_{Y}}{\sigma_{Y}}\right) d Y_{k}\right) \\
& \times\left(\sum_{r=0}^{\infty} A_{r 0} \varphi_{r}^{(1)}\left(X_{k}\right)\right)^{-1}
\end{aligned}
$$




$$
\begin{aligned}
& =\exp \left\{\frac{\left(C \mu_{Y}+l \sigma_{Y}^{2}\right)^{2}}{2 \sigma_{Y}^{2} C^{2}}-\frac{\left(C \mu_{Y}^{2}+2 l \sigma_{Y}^{2} K\right)}{2 \sigma_{Y}^{2} C}\right\} \\
& \quad \times\left(\int_{-\infty}^{\infty} N\left(Y_{k} ; \xi_{k}, \sigma_{Y}^{2}\right)\right. \\
& \left.\quad \times \sum_{r=0}^{\infty} \sum_{s=0}^{\infty} A_{r s} \varphi_{r}^{(1)}\left(X_{k}\right) \frac{1}{\sqrt{s !}} H_{s}\left(\frac{Y_{k}-\mu_{Y}}{\sigma_{Y}}\right) d Y_{k}\right) \\
& \quad \times\left(\sum_{r=0}^{\infty} A_{r 0} \varphi_{r}^{(1)}\left(X_{k}\right)\right)^{-1},
\end{aligned}
$$

where

$$
\xi_{k} \equiv \frac{1}{C}\left(C \mu_{Y}+l \sigma_{Y}^{2}\right)
$$

and $d_{s 0}^{(l)}$ are coefficients satisfying the following relationship:

$$
H_{s}\left(\frac{Y_{k}-\mu_{Y}}{\sigma_{Y}}\right)=\sum_{j=0}^{s} d_{s j}^{(l)} H_{j}\left(\frac{Y_{k}-\xi_{k}}{\sigma_{Y}}\right) .
$$

By using the orthonormal condition of Hermite polynomial,

$$
\begin{aligned}
\int_{-\infty}^{\infty} & N\left(Y_{k} ; \xi_{k}, \sigma_{Y}^{2}\right) \frac{1}{\sqrt{i !}} H_{i}\left(\frac{Y_{k}-\xi_{k}}{\sigma_{Y}}\right) \frac{1}{\sqrt{j !}} H_{j}\left(\frac{Y_{k}-\xi_{k}}{\sigma_{Y}}\right) d Y_{k} \\
& =\delta_{i j},
\end{aligned}
$$

the function $I_{l}$ in (21) can be calculated as

$$
\begin{aligned}
I_{l}= & \exp \left\{\frac{\left(C \mu_{Y}+l \sigma_{Y}^{2}\right)^{2}}{2 \sigma_{Y}^{2} C^{2}}-\frac{C \mu_{Y}^{2}+2 l \sigma_{Y}^{2} K}{2 \sigma_{Y}^{2} C}\right\} \\
& \times \frac{\sum_{r=0}^{\infty} \sum_{s=0}^{\infty} A_{r s} \varphi_{r}^{(1)}\left(X_{k}\right)\left(d_{s 0}^{(l)} / \sqrt{s !}\right)}{\sum_{r=0}^{\infty} A_{r 0} \varphi_{r}^{(1)}\left(X_{k}\right)} .
\end{aligned}
$$

Therefore, (20) can be expressed as follows:

$$
\begin{gathered}
\left\langle y_{k} \mid Z_{k-1}\right\rangle=\exp \left\{\frac{\left(C \mu_{Y}+\sigma_{Y}^{2}\right)^{2}}{2 \sigma_{Y}^{2} C^{2}}-\frac{C \mu_{Y}^{2}+2 \sigma_{Y}^{2} K}{2 \sigma_{Y}^{2} C}\right\} \\
\times \frac{\sum_{s=0}^{\infty} \mathbf{A}_{(s), k} \Phi\left(X_{k}\right)\left(d_{s 0}^{(1)} / \sqrt{s !}\right)}{\sum_{r=0}^{\infty} A_{r 0} \varphi_{r}^{(1)}\left(X_{k}\right)}, \\
\left\langle\left(y_{k}-y_{k}^{*}\right)^{2} \mid Z_{k-1}\right\rangle \\
=\exp \left\{\frac{\left(C \mu_{Y}+2 \sigma_{Y}^{2}\right)^{2}}{2 \sigma_{Y}^{2} C^{2}}-\frac{C \mu_{Y}^{2}+4 \sigma_{Y}^{2} K}{2 \sigma_{Y}^{2} C}\right\} \\
\times \frac{\sum_{s=0}^{\infty} \mathbf{A}_{(s), k} \Phi\left(X_{k}\right)\left(d_{s 0}^{(2)} / \sqrt{s !}\right)}{\sum_{r=0}^{\infty} A_{r 0} \varphi_{r}^{(1)}\left(X_{k}\right)}-y_{k}^{*},
\end{gathered}
$$

with

$$
\begin{gathered}
\Phi\left(X_{k}\right) \equiv\left(\varphi_{0}^{(1)}\left(X_{k}\right), \varphi_{1}^{(1)}\left(X_{k}\right), \ldots\right)^{t} \\
\mathbf{A}_{(s), k} \equiv \mathbf{a}_{(s), k}^{*}, \quad(s=1,2, \ldots), \\
\mathbf{A}_{(0), k} \equiv\left(A_{00}, A_{10}, A_{20}, \ldots\right), \\
\mathbf{a}_{(s), k}^{*} \equiv\left\langle\mathbf{a}_{(s), k} \mid Z_{k-1}\right\rangle,
\end{gathered}
$$

where $t$ denotes the transpose of a matrix. Furthermore, using the definition of Laguerre polynomial and (1), the expansion coefficient $B_{\mathbf{m} n}$ can be calculated as follows:

$$
\begin{aligned}
& B_{\mathbf{m} n}=\sqrt{\frac{\Gamma\left(m_{k}^{*}\right) n !}{\Gamma\left(m_{k}^{*}+n\right)}} \sum_{s_{1}=0}^{n}(-1)^{s_{1}}\left(\begin{array}{l}
n \\
s_{1}
\end{array}\right) \frac{1}{n !} \frac{\Gamma\left(m_{k}^{*}+n\right)}{\Gamma\left(m_{k}^{*}+s_{1}\right)}\left(\frac{1}{s_{k}^{*}}\right)^{s_{1}} \\
& \times \sum_{s_{2}=0}^{s_{1}}\left(\begin{array}{l}
s_{1} \\
s_{2}
\end{array}\right) \exp \left\{\frac{\left(C \mu_{Y}+s_{2} \sigma_{Y}^{2}\right)^{2}}{2 \sigma_{Y}^{2} C^{2}}-\frac{C \mu_{Y}^{2}+2 s_{2} \sigma_{Y}^{2} K}{2 \sigma_{Y}^{2} C}\right\} \\
& \times\left\langle v_{k}^{s_{1}-s_{2}}\right\rangle \\
& \times \frac{\sum_{s=0}^{\infty}\left\langle\theta_{\mathbf{m}}^{(1)}\left(\mathbf{a}_{k}\right) \mathbf{a}_{(s), k} \mid Z_{k-1}\right\rangle \Phi\left(X_{k}\right)(1 / \sqrt{s !}) d_{s 0}^{\left(s_{2}\right)}}{\sum_{r=0}^{\infty} A_{r 0} \varphi_{r}^{(1)}\left(X_{k}\right)} .
\end{aligned}
$$

From (19) and (26)-(28), it can be found that the parameters $z_{k}^{*}, \Omega_{k}$ and the expansion coefficient $B_{\mathbf{m} n}$ are given by the predictions of unknown parameter $\mathbf{a}_{k}$, the statistics of the external noise $v_{k}$, and the input observations $X_{k}$.

By considering (11), the prediction to perform the recurrence estimation can be given for an arbitrary polynomial function $g_{\mathbf{N}}\left(\mathbf{a}_{k+1}\right)$ with $\mathbf{N t h}$ order of $\mathbf{a}_{k+1}$ can be expressed as

$$
g_{\mathbf{N}}^{*}\left(\mathbf{a}_{k+1}\right) \equiv\left\langle g_{\mathbf{N}}\left(\mathbf{a}_{k+1}\right) \mid Z_{k}\right\rangle=\left\langle g_{\mathbf{N}}\left(\mathbf{a}_{k}\right) \mid Z_{k}\right\rangle=\widehat{g}_{\mathbf{N}}\left(\mathbf{a}_{k}\right) .
$$

2.3. Prediction of Output Probability Distribution for Sound Environment System. Because the conditional probability density function $P(Y \mid X)$ can be considered as an invariant system characteristic, reflecting mainly the proper correlation relationship between the two sound pressure levels $X$ and $Y$ in the sound environment system, the output probability distribution $P_{s}(Y)$ in decibel scale can be predicted, as $P_{s}(Y)=$ $\langle P(Y \mid X)\rangle_{X}$. Thus, based on (1) and using the estimated parameter $\widehat{\mathbf{a}}_{k} \equiv\left(\widehat{A}_{11}, \widehat{A}_{12}, \ldots\right)$, the output probability density function $P_{s}(Y)$ can be predicted from the observed input data $X$, as follows:

$$
P_{s}(Y)=P_{0}(Y) \sum_{s=0}^{\infty}\left\langle\frac{\sum_{r=0}^{\infty} \widehat{A}_{r s} \varphi_{r}^{(1)}(X)}{\sum_{r=0}^{\infty} A_{r 0} \varphi_{r}^{(1)}(X)}\right\rangle_{X} \varphi_{s}^{(2)}(Y) .
$$

\section{Application to Real Sound Environment System}

3.1. Application to Sound Insulation System. In order to confirm the effectiveness of the proposed method, it was 
TABLE 1: Statistics of the input, output signals and the background noise in sound insulation system.

\begin{tabular}{lcc}
\hline & Mean $\left[\mathrm{W} / \mathrm{m}^{2}\right]$ & Standard deviation $\left[\mathrm{W} / \mathrm{m}^{2}\right]$ \\
\hline Input signal & $1.3981 \times 10^{-4}$ & $8.2881 \times 10^{-5}$ \\
Output signal & $1.0633 \times 10^{-6}$ & $5.0934 \times 10^{-6}$ \\
Background noise & $1.0633 \times 10^{-6}$ & $4.4550 \times 10^{-6}$ \\
\hline
\end{tabular}

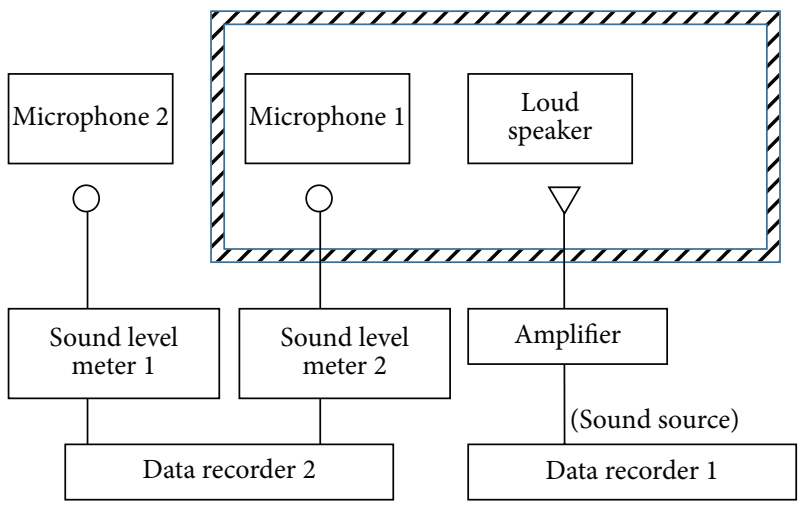

FIGURE 1: A schematic drawing of the experimental setup in sound insulation system.

applied to real data observed in a sound environment system. Acoustic signals observed by two microphones in indoors and outdoors for a house were adopted as input and output data for the sound insulation system. The schematic drawing of the sound environment system is shown in Figure 1. The rock music was selected as an input signal by considering the aggravation of "Karaoke" noise pollution problem, and white noise was adopted as a background noise. The statistics of the input, output signals and the background noise are shown in Table 1 . The input and output fluctuation data simultaneously measured with every sampling interval $1 \mathrm{~s}$. Based on the 500 data, the expansion coefficients $A_{r s}$ in (1) were estimated on the basis of the input signal $X_{k}$ and observation $z_{k}$ under existence of the background noise.

Based on the estimated expansion coefficients, the output response probability distribution excited by an arbitrary input signal was predicted. The 200 sampled data following the data used for the evaluation of expansion coefficients were adopted for predicting the output response probability distribution. Figure 2 shows the comparison between theoretically predicted curves and experimentally sampled points on the output probability distribution. The cumulative distributions of sound level are shown in this figure. The "theoretical curves" in this figure were obtained by predicting the probability density function of the output level $Y$ based on the observed data of the input level $X$ by use of the theoretical expression. In this figure, "1st-3rd approximations" considered the expansion coefficients $\widehat{A}_{11}, \widehat{A}_{12}, \widehat{A}_{21}$, and $\widehat{A}_{22}$ successively in (30). The "experimental values" represent the frequency distributions obtained directly from the observed data of the output level $Y$.

For comparison, the prediction results of the output probability distribution by introducing the standard regression

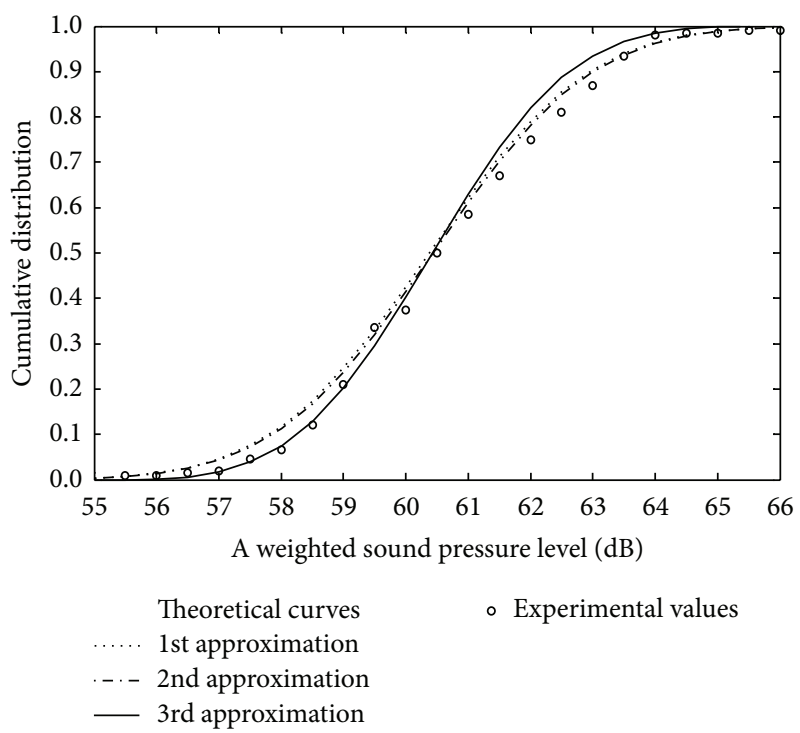

FIGURE 2: Comparison between experimentally sampled values and theoretically predicted curves by the proposed method on the output probability distribution for the sound insulation system.

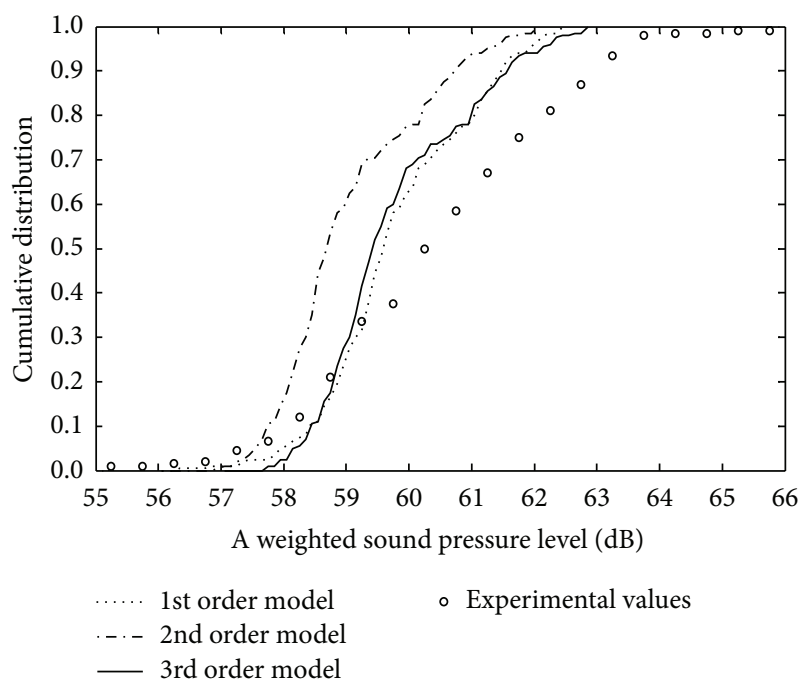

FIgURE 3: Comparison between experimentally sampled values and theoretically predicted curves by the standard regression models on the output probability distribution for the sound insulation system.

models described by the following equations are shown in Figure 3:

$$
\begin{gathered}
\widehat{Y}=a_{1}+b_{1} X \quad(1 \text { st order model }) \\
\widehat{Y}=a_{2}+b_{2} X+c_{2} X^{2} \quad(2 \text { nd order model }) \\
\widehat{Y}=a_{3}+b_{3} X+c_{3} X^{2}+d_{3} X^{3} \quad(3 \text { rd order model }) .
\end{gathered}
$$


TABLE 2: Comparison between the experimental values and theoretically predicted values for several noise evaluation quantities in $\mathrm{dB}$ evaluated from Figures 2 and 3.

\begin{tabular}{lccccc}
\hline $\begin{array}{l}\text { Noise evaluation } \\
\text { quantities }\end{array}$ & $L_{5}$ & $L_{10}$ & $L_{50}$ & $L_{90}$ & $L_{95}$ \\
\hline $\begin{array}{l}\text { Experimental } \\
\text { values }\end{array}$ & 63.6 & 63.1 & 60.5 & 58.2 & 57.6 \\
$\begin{array}{l}\text { Theoretical curve } \\
\text { (1st } \\
\text { approximation) }\end{array}$ & 63.7 & 63.0 & 60.4 & 57.7 & 57.1 \\
$\begin{array}{l}\text { Theoretical curve } \\
\text { (2nd }\end{array}$ & 63.7 & 63.0 & 60.5 & 57.7 & 57.1 \\
$\begin{array}{l}\text { approximation) } \\
\text { Theoretical curve } \\
\text { (3rd }\end{array}$ & 63.2 & 62.5 & 60.5 & 58.2 & 57.7 \\
$\begin{array}{l}\text { approximation) } \\
\text { Regression model } \\
\text { (1st order model) }\end{array}$ & 61.9 & 61.5 & 59.6 & 58.4 & 57.9 \\
$\begin{array}{l}\text { Regression model } \\
\text { (2nd order model) }\end{array}$ & 61.2 & 60.7 & 58.7 & 57.7 & 57.5 \\
$\begin{array}{l}\text { Regression model } \\
\text { (3rd order model) }\end{array}$ & 62.1 & 61.6 & 59.4 & 58.4 & 58.1 \\
\hline
\end{tabular}

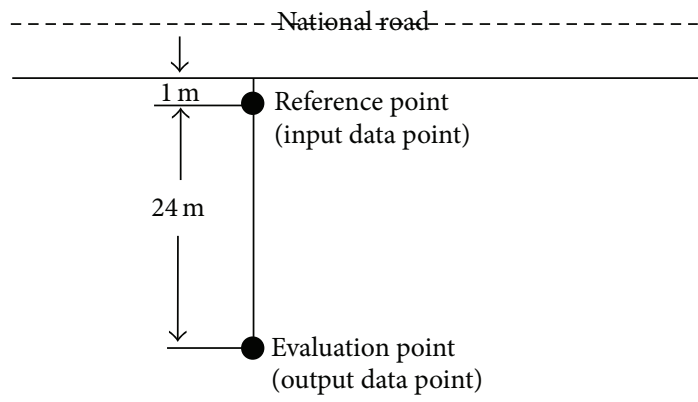

FIgURE 4: A schematic drawing of the experiment in road traffic noise environment near a national road.

The regression coefficients $a_{i}, b_{i}, c_{i}$, and $d_{i}$ in (31) were determined by applying the extended Kalman filter [5] after introducing the following observation equation:

$$
z_{k}=10^{Y_{k} / 10-12}+v_{k} \text {. }
$$

The theoretically predicted curves based on the proposed method show better agreement with the experimentally sampled values than the results by applying the extended Kalman filter based on the standard regression models.

From the cumulative distributions in Figures 2 and 3, noise evaluation quantities $L_{x}((100-x)$ percentile level $)$ can be evaluated. Several noise evaluation quantities obtained from these figures are shown in Table 2. It is obvious that the proposed method provides a more accurate prediction than the results based on the standard regression models.

3.2. Application to Road Traffic Noise Environment. The effectiveness of the proposed method was confirmed experimentally by applying it to real road traffic noise data observed in the complicated sound environment near a national road.
TABLE 3: Statistics of the input, output signals and the background noise in road traffic noise environment.

\begin{tabular}{lcc}
\hline & Mean $\left[\mathrm{W} / \mathrm{m}^{2}\right]$ & Standard deviation $\left[\mathrm{W} / \mathrm{m}^{2}\right]$ \\
\hline Input signal & $2.7352 \times 10^{-5}$ & $3.3987 \times 10^{-5}$ \\
Output signal & $1.7185 \times 10^{-6}$ & $2.0577 \times 10^{-6}$ \\
Background noise & $1.7185 \times 10^{-6}$ & $2.1789 \times 10^{-6}$ \\
\hline
\end{tabular}

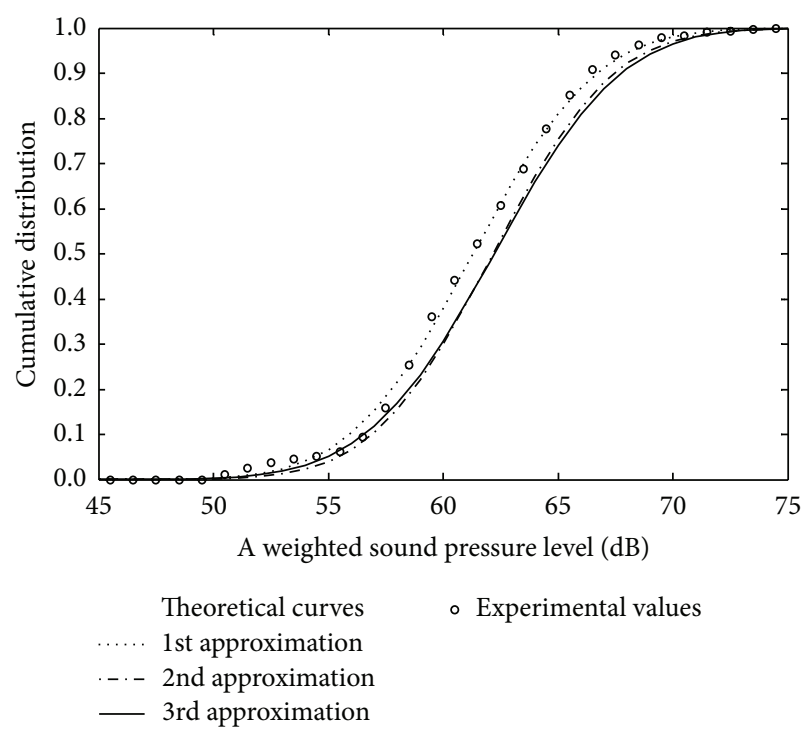

Figure 5: Comparison between experimentally sampled values and theoretically predicted curves by the proposed method on the probability distribution at the evaluation point for the road traffic noise environment near a national road.

In order to evaluate the sound environment around the main line, the sound level at an evaluation point has to be predicted on the basis of the observation at a reference point. After regarding the sound levels at a reference point and an evaluation point as system input $X$ and output $Y$, respectively, the probability distribution in decibel scale at the evaluation point connected with several evaluation quantities of the sound environment was predicted on the basis of the observation at the reference point. The reference point and the evaluation point were chosen at the positions being $1 \mathrm{~m}$ and $25 \mathrm{~m}$ apart from one side of the road as shown in Figure 4. The statistics of the input, output signals and the background noise are shown in Table 3. By applying the proposed method, the probability density function of the sound level at the evaluation point was predicted on the basis of the observation at the reference point. Road traffic noise was measured by the use of the sound level meter at every $0.2 \mathrm{~s}$. Through the same procedure in Section 3.1, the expansion coefficients $A_{r s}$ in (1) were first estimated.

Based on the estimates of the expansion coefficients, the probability density function of road traffic noise at the evaluation point was predicted by measuring the road traffic noise data at the reference point. The predicted results are shown in Figures 5 and 6. Several noise evaluation quantities evaluated from Figures 5 and 6 are shown in Table 4. From these results, it can be seen that the theoretically predicted 


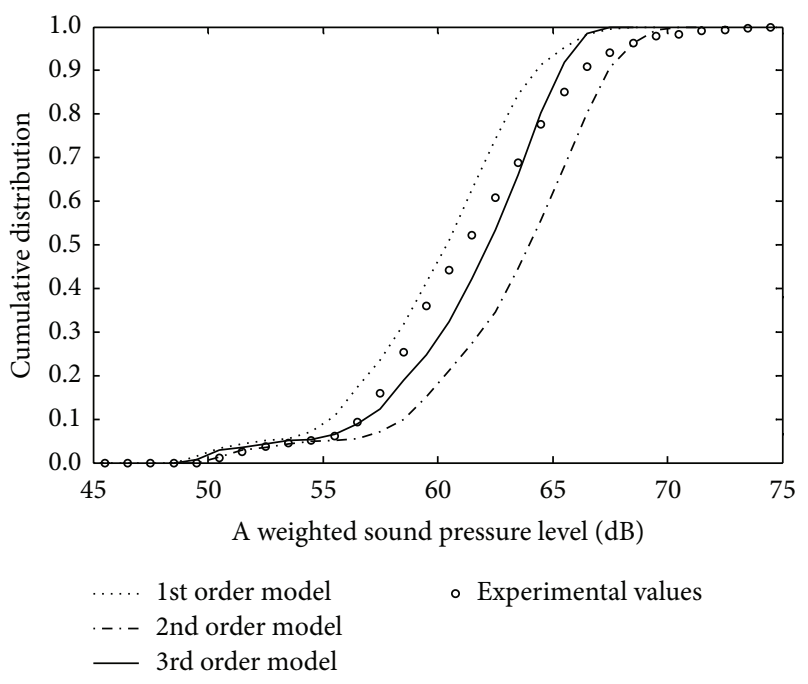

Figure 6: Comparison between experimentally sampled values and theoretically predicted curves by the standard regression models on the probability distribution at the evaluation point for the road traffic noise environment near a national road.

TABLE 4: Comparison between the experimental values and theoretically predicted values for several noise evaluation quantities in $\mathrm{dB}$ evaluated from Figures 5 and 6.

\begin{tabular}{lccccc}
\hline $\begin{array}{l}\text { Noise evaluation } \\
\text { quantities }\end{array}$ & $L_{5}$ & $L_{10}$ & $L_{50}$ & $L_{90}$ & $L_{95}$ \\
\hline $\begin{array}{l}\text { Experimental } \\
\text { values }\end{array}$ & 68.0 & 66.3 & 61.3 & 56.6 & 54.5 \\
$\begin{array}{l}\text { Theoretical curve } \\
\text { (1st }\end{array}$ & 68.1 & 66.7 & 61.3 & 55.8 & 54.5 \\
$\begin{array}{l}\text { approximation) } \\
\begin{array}{l}\text { Theoretical curve } \\
\text { (2nd }\end{array}\end{array}$ & 68.9 & 67.4 & 62.2 & 56.9 & 55.4 \\
$\begin{array}{l}\text { approximation) } \\
\begin{array}{l}\text { Theoretical curve } \\
\text { (3rd }\end{array}\end{array}$ & 69.3 & 67.7 & 62.2 & 56.5 & 55.0 \\
$\begin{array}{l}\text { approximation) } \\
\text { Regression model } \\
\text { (1st order model) }\end{array}$ & 65.6 & 64.4 & 60.4 & 55.2 & 52.8 \\
$\begin{array}{l}\text { Regression model } \\
\text { (2nd order model) }\end{array}$ & 68.4 & 67.5 & 64.1 & 58.4 & 55.0 \\
$\begin{array}{l}\text { Regression model } \\
\text { (3rd order model) }\end{array}$ & 66.0 & 65.4 & 62.2 & 56.7 & 53.7 \\
\hline
\end{tabular}

values by use of the proposed method show good agreement with the experimental values, as compared with the results applying the extended Kalman filter for the standard regression models.

\section{Conclusions}

In this paper, an evaluation method of complex sound environment systems under existence of an external noise has been proposed. More specifically, by paying attention to the energy variables satisfying the additive property of the specific signal and the external noise, a method for estimating the correlation information between the input and output variables has been theoretically derived on the basis of the observations contaminated by the external noise. Furthermore, a prediction method of the output probability distribution in decibel scale has been derived based on the observations of the input level. The proposed prediction method has been realized by introducing a sound environment model of the conditional probability type in decibel scale. The proposed method has then been applied to the estimation and prediction of a real sound insulation system and road traffic noise environment, and it has been experimentally verified that good results have been achieved with this method.

The proposed stochastic signal processing method is quite different from the traditional standard approach. However, it is still at its early stage of study, and there are a number of practical problems to be explored in the future, starting from the result of the basic study in this paper. Some of the problems are the following.

(i) The proposed method should be applied to real prediction problems of output probability distribution for many other sound environment systems, and its practical usefulness should be verified in each real situation.

(ii) The theory should be extended to further practical cases with multi-input and multioutput systems.

(iii) An optimal number of expansion terms in the proposed stochastic signal processing method of expansion expression type should be found.

\section{Appendix}

\section{Derivation of the Estimate}

The conditional joint probability density function of the parameter $\mathbf{a}_{k}$ and the observation $z_{k}$ can be generally expanded in a statistical orthogonal expansion series:

$$
\begin{aligned}
P\left(\mathbf{a}_{k}, z_{k} \mid Z_{k-1}\right)= & P_{0}\left(\mathbf{a}_{k} \mid Z_{k-1}\right) P_{0}\left(z_{k} \mid Z_{k-1}\right) \\
& \times \sum_{\mathbf{m}=\mathbf{0}}^{\infty} \sum_{n=0}^{\infty} B_{\mathbf{m} n} \theta_{\mathbf{m}}^{(1)}\left(\mathbf{a}_{k}\right) \theta_{n}^{(2)}\left(z_{k}\right) .
\end{aligned}
$$

After substituting (A.1) into (12), taking the conditional expectation of the function $f_{\mathbf{M}}\left(\mathbf{a}_{k}\right)$, and using the orthonormal condition for the function $\theta_{\mathbf{m}}^{(1)}\left(\mathbf{a}_{k}\right)$, (13) can be derived.

\section{Conflict of Interests}

The authors declare that there is no conflict of interests regarding the publication of this paper. 


\section{References}

[1] A. Ikuta, H. Masuike, and M. Ohta, "A digital filter for stochastic systems with unknown structure and its application to psychological evaluation of sound environment," IEICE Transactions on Information and Systems, vol. 88, no. 7, pp. 1519-1525, 2005.

[2] M. Ohta and A. Ikuta, "Acoustic signal processing for generalized regression analysis with reduced information loss based on data observed with an amplitude limitation," Acustica, vol. 81, no. 2, pp. 129-135, 1995.

[3] M. Ohta and T. Koizumi, "General statistical treatment of the response of a nonlinear rectifying device to a stationary random input," IEEE Transactions on Information Theory, vol. 14, no. 4, pp. 595-598, 1968.

[4] M. Ohta and H. Yamada, "New methodological trials of dynamical state estimation for the noise and vibration environmental system-establishment of general theory and its application to urban noise problems," Acustica, vol. 55, no. 4, pp. 199-212, 1984.

[5] H. J. Kushner, "Approximations to optimal nonlinear filters," IEEE Transactions on Automatic Control, vol. AC-12, no. 5, pp. 546-556, 1967. 


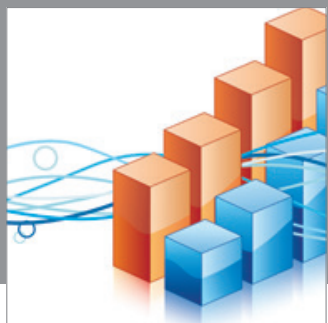

Advances in

Operations Research

mansans

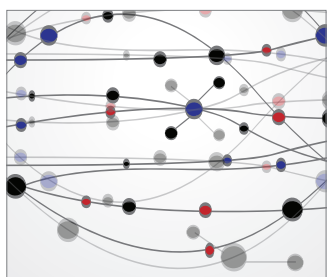

The Scientific World Journal
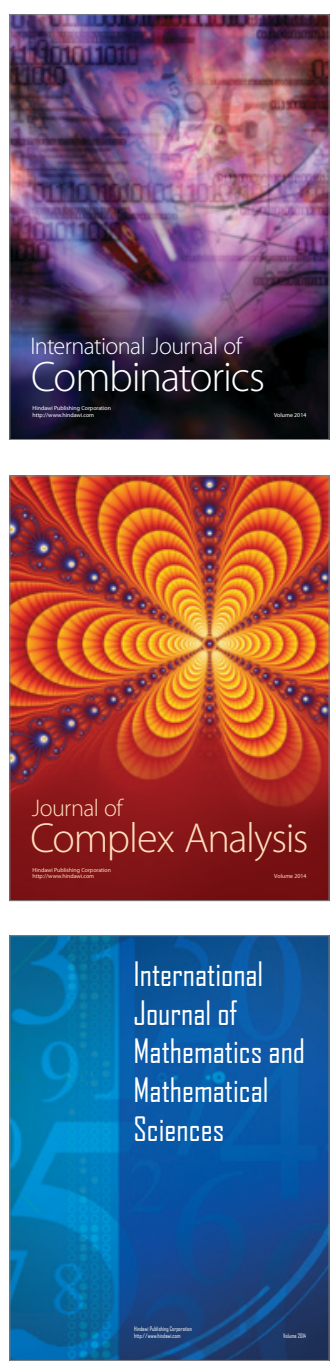
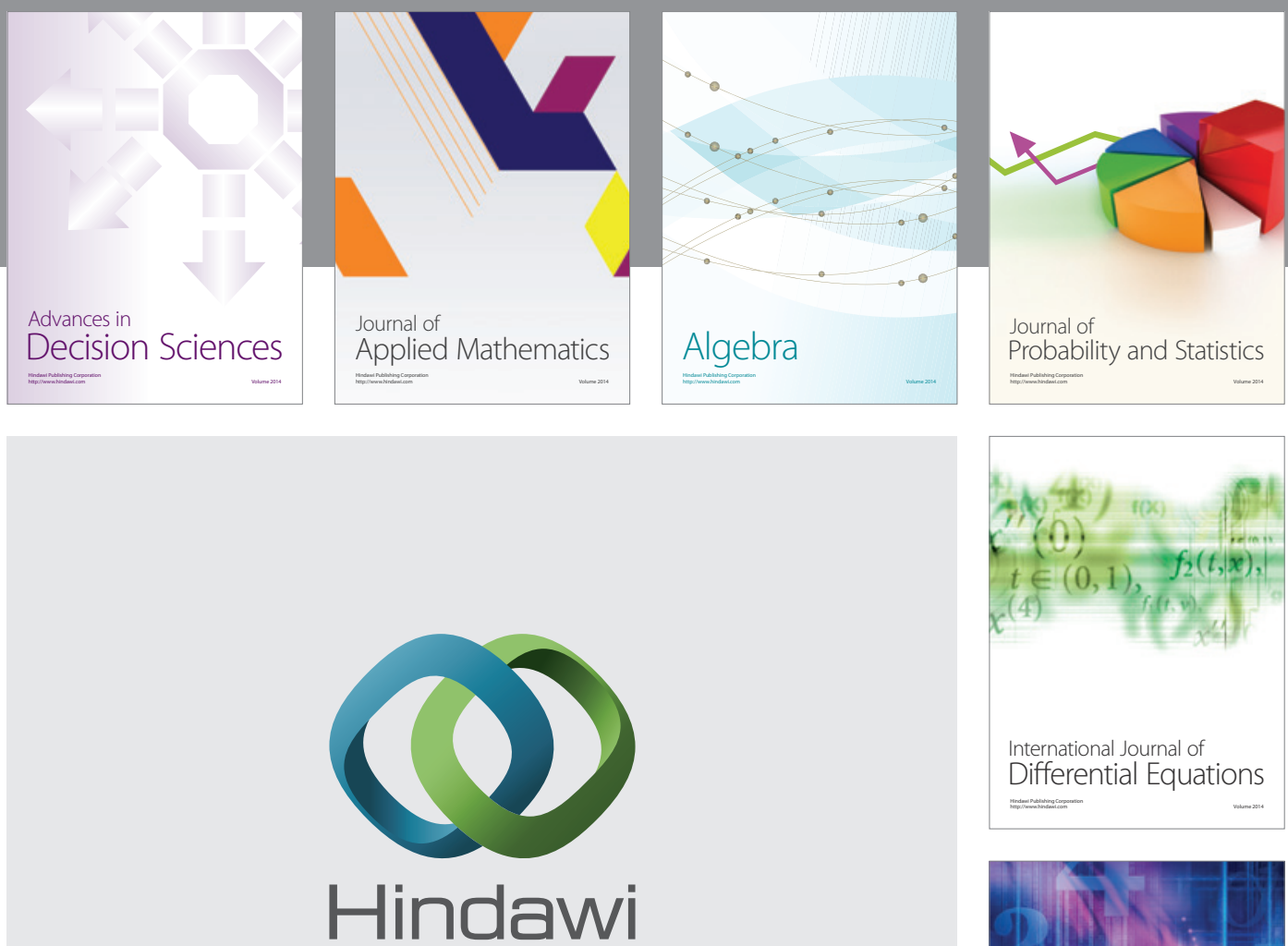

Submit your manuscripts at http://www.hindawi.com
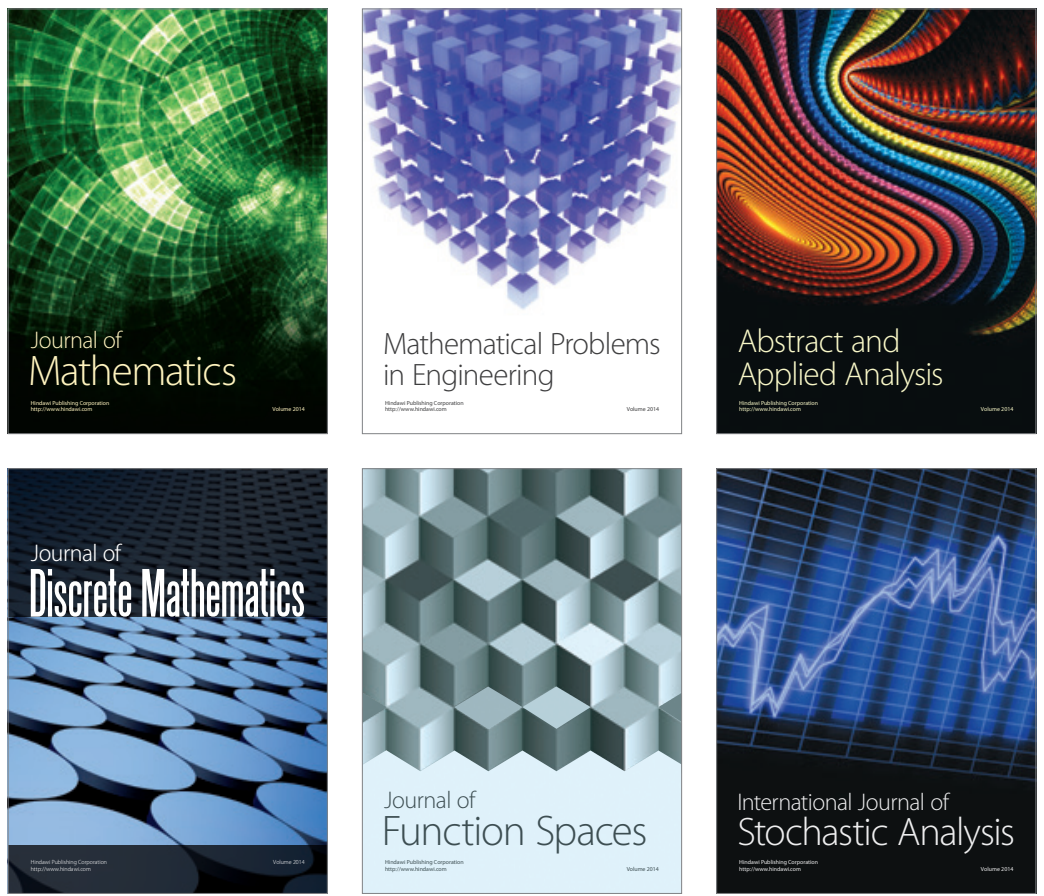

Journal of

Function Spaces

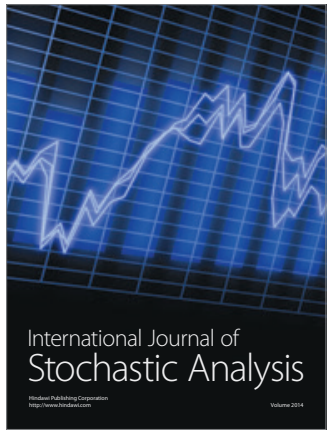

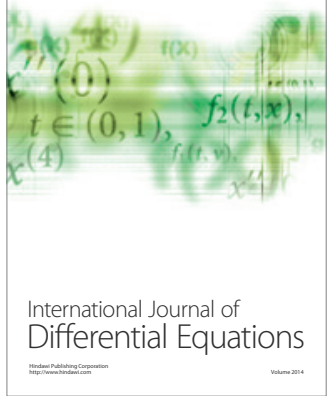
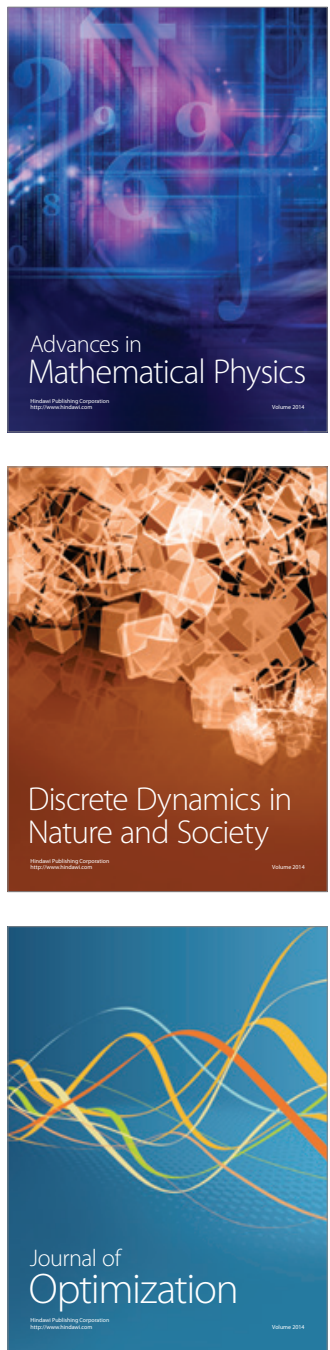